\title{
Brown urine and black hip
}

\section{Nikiforos Galanis MD PhD, Margaritis Kyrkos MD PhD}

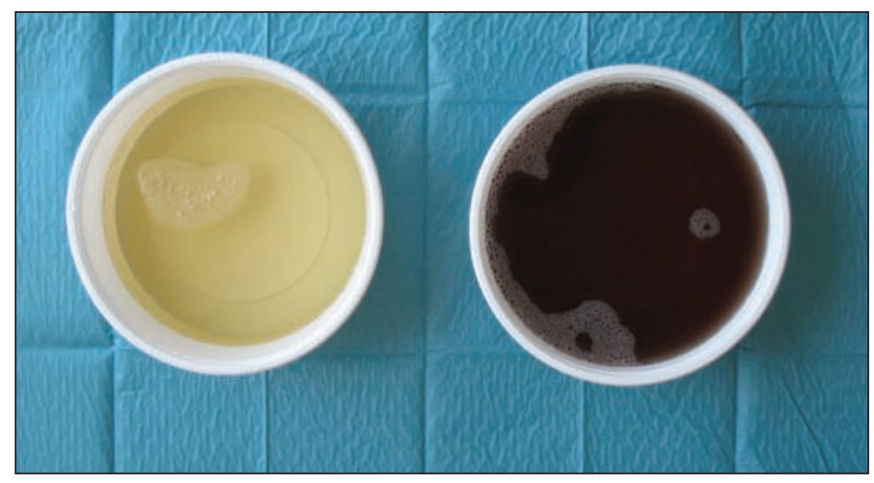

Figure 1: Urine samples immediately after discharge (left) and one to two hours after exposure to air (right).

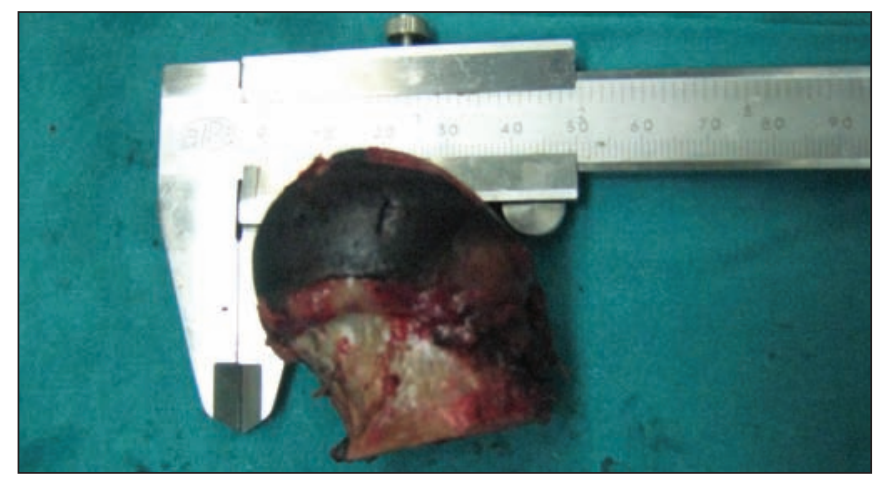

Figure 2: Black appearance of the femoral head due to the pigmented cartilage.
Competing interests: None declared.

This article has been peer reviewed.

Affiliations: From the Medical School, Aristotle University of Thessaloniki, and the Department of Orthopaedics, Papageorgiou General Hospital, Thessaloniki, Greece

Correspondence to: Nikiforos Galanis, kyros@med.auth.gr

CMAJ 2012. DOI:10.1503 /cmaj.111916
A 69-year-old woman presented with rapidly progressive pain in her right hip over the last two months. Physical examination revealed a restricted range of motion associated with pain. Brownish pigmentation was noticed on her sclerae (Appendix 1, available at www.cmaj.ca/lookup/suppl/doi:10 $.1503 /$ cmaj.111916/-/DC1), ears, oral mucosa, nails and vulvar vestibule. When a urine sample was taken, the urine initially was of normal colour but gradually became dark brown on exposure to air after several hours (Figure 1). Radiographic images and magnetic resonance imaging showed osteonecrosis of the right femoral head. Biopsy of a skin sample from one of her ears was consistent with ochronosis, a condition in which bluish-black pigment is progressively deposited in connective tissues. The patient underwent a successful total hip arthroplasty. During surgery, the hip appeared black because of the pigmented cartilage of the femoral head (Figure 2). The histologic features of the surgical specimen were compatible with ochronosis and osteonecrosis. The presence of ochronosis and the darkening of the urine due to excess levels of homogentisic acid confirmed the diagnosis of alkaptonuria. The patient was asymptomatic after follow-up for two years.

Alkaptonuria is a rare autosomal recessive metabolic disease in which the homogentisate 1,2dioxygenase enzyme is absent, resulting in raised blood levels of homogentisic acid. ${ }^{1}$ The estimated prevalence is as low as 1 per 25000 in some populations and is affected by factors such as genetic isolation and consanguinity. ${ }^{2}$ Ochronosis-related arthropathy, including lumbosacral ankylosis, and discoloration of the urine due to elevated levels of homogentisic acid constitute the main manifestations of alkaptonuria in adults. Less common clinical features include calcification of the aortic valve and renal dysfunction associated with renal stones. Children are typically asymptomatic; alkaptonuria should be suspected if there is a history of dark urine, or stained diapers or underclothes. In such instances, measurement of excess levels of homogentisic acid in urine will confirm the diagnosis. No effective therapy exists. Nitisinone, a medication found to inhibit the production of homogentisic acid, is being evaluated as a possible treatment; further investigation is required. ${ }^{3}$

\section{References}

1. Phornphutkul C, Introne WJ, Perry MB, et al. Natural history of alkaptonuria. $N$ Engl J Med 2002;347:2111-21.

2. Al-Sbou M, Mwafi N, Lubad MA. Identification of forty cases with alkaptonuria in one village in Jordan. Rheumatol Int 2011 Nov. 16 [Epub ahead of print].

3. Introne WJ, Perry MB, Troendle J, et al. A 3-year randomized therapeutic trial of nitisinone in alkaptonuria. Mol Genet Metab 2011;103:307-14. 